\title{
The narrow band AOTF based hyperspectral microscopic imaging on the rat skin stratum configuration
}

\section{Zhang}

\section{H. Wang}

haowang@fjnu.edu.cn

\section{J. Huang}

Q. Gao

\begin{abstract}
Fujian Provincial Key Laboratory for Photonics Technology, Key Laboratory of Optoelectronic Science and Technology for Medicine of Ministry of Education, College of Photonic and Electronic Engineering, Fujian Normal University, Fuzhou 350007, People's Republic of China

Fujian Provincial Key Laboratory for Photonics Technology, Key Laboratory of Optoelectronic Science and Technology for Medicine of Ministry of Education, College of Photonic and Electronic Engineering, Fujian Normal University, Fuzhou 350007, People's Republic of China
\end{abstract}

Fujian Provincial Key Laboratory for Photonics Technology, Key Laboratory of Optoelectronic Science and Technology for Medicine of Ministry of Education, College of Photonic and Electronic Engineering, Fujian Normal University, Fuzhou 350007, People's Republic of China

Fujian Provincial Key Laboratory for Photonics Technology, Key Laboratory of Optoelectronic Science and Technology for Medicine of Ministry of Education, College of Photonic and Electronic Engineering, Fujian Normal University, Fuzhou 350007, People's Republic of China

A noncollinear acousto-optic tunable filter (AOTF) was designed with a comprehensive treatment of the properties of $\mathrm{TeO}_{2}$ as an acoustooptic (A-0) material. The results of optical testing validated that it predicted the performance of the designed AOTF. The bandwidth of the AOTF was very narrow in the visible light range. The high spectral resolution of AOTF was useful in practical applications to the hyperspectral imaging. The experimentally observed spectral pattern of the diffracted light was nearly the same as the theoretical result. The measured tuning relationship between the diffracted central optical wavelength and acoustic frequency was in accordance with the theoretical prediction. It demonstrates the accuracy of our design theory. Furthermore, by selecting the AOTF as the spectroscopic element, a hyperspectral microscopic imaging system was designed. The hyperspectral microscopic images of the rat skin tissue under the different optical center wavelength were acquired. The experimental results indicated that the imaging performance was satisfactory. The stratums of the rat skin can be clearly distinguished. The inner details of the epidermis and the corium can be shown on the hyperspectral microscopic images. Some differences also can be found by the comparison of the hyperspectal images under the different optical wavelengths. The study indicated the applicability and the advantage of our system on biomedicine area.

[DOI: http://dx.doi.org/10.2971/jeos.2014.14034]

Keywords: Hyperspectral imaging, biomedicine, acousto-optic tunable filter, skin

\section{INTRODUCTION}

AOTF is a kind of all solid state optical filter with fast scan speed. Its function is based on the acousto-optic interaction in the birefringent material and possesses many attractive features: real-time electronic tuning with wide tuning range, large throughput etc. The AOTF had been widely used in the tuning dye lasers, optical calculation, spectral analysis, hyperspectral imaging and other related areas [1]-[6]. Although many kinds of AOTF (based on the crystal or optical fiber) were commercial available, scientists have been working hard on developing an AOTF with better performance. Generally, the narrower selected spectral bandwidth, satisfactory image resolution, little beam shift with the tuning central optical wavelength of the diffracted light were the focus of the design. The perfect performance of an AOTF is of significance for keeping the good detection ability in the related applications. In these years, the $\mathrm{TeO}_{2}$ based noncollinear AOTF have been more and more attractive for the demonstrated good performance (high diffraction efficiency, large angular aperture, and etc.). In many previous works of the AOTF design, some obvious error had been introduced for neglecting certain material property of A-O crystal [7, 8]. In our foregoing studies, an accurate design theory considering the birefringence and the rotatory property of $\mathrm{TeO}_{2}$ crystal was put forward. And it was approved that our theory was much functional in ensuring the design accuracy. Therefore, we designed a $\mathrm{TeO}_{2}$ based AOTF, and the main indices were experimentally measured. In the visible range, the experimental results illuminated the good performance of the designed AOTF. Besides, the experimental parameters were in accordance with the theoretical prodiction. For expressing the feasibility of the AOTF in the biomedical application, an AOTF based hyperspectral imaging system was set up. The rat skin tissue was selected, and its hyperspectral microscopic images at s series of optical wavelengths were collected by running the system. The imaging performance was satisfactory. The inner configuration was distinguishable from the acquired microscopic images. 


\section{THE ACCURATE ACOUSTO-OPTIC THEORY}

About the design of noncollinear AOTF, the previous A-O interaction theories had introduced certain error by neglecting the related material property of $\mathrm{TeO}_{2}$ crystal. It had decreased the accuracy of AOTF design to some extent $[7,8]$. In our A-O theory, the birefringence and the rotatory property were two indispensable conditions for consideration. And our theory had been identified effective of improving the design accuracy and the performance of the noncollinear AOTF. Figure 1 gave the wave vector diagram of A-O interaction. The two eigen wave modes propagating in $\mathrm{TeO}_{2}$ were right-handed elliptical polarized mode and left-handed elliptical polarized mode. The incident light and the diffracted one were on the different eigen wave modes. The birefringence of $\mathrm{TeO}_{2}$ crystal ensured that the wavevectors under the two eigen modes were unequal.

As the parallel tangents momentum-matching condition was satisfied, the incident optical wave vector $k_{i}$, the diffracted optical wave vector $k_{d}$ and the acoustic wave vector $k_{a}$ fulfilled the relationship of $k_{i}+k_{a}=k_{d}$. The tangents of the loci of incident and diffracted wave vectors were parallel. In the direction of the optical axis, the difference of wavevector is related with a factor $\sigma$-it expressed the degree of the rotatory property of the $\mathrm{TeO}_{2}$ crystal).

The refractive indices of the incident beam $\left(n_{i}\right)$ and the diffracted beam $\left(n_{d}\right)$ were

$$
\begin{aligned}
& n_{i}=\left[\frac{\cos ^{2} \theta_{i}}{n_{o}^{2}(1+\sigma)^{2}}+\frac{\sin ^{2} \theta_{i}}{n_{e}^{2}}\right]^{-1 / 2} \\
& n_{d}=\left[\frac{\cos ^{2} \theta_{d}}{n_{o}^{2}(1-\sigma)^{2}}+\frac{\sin ^{2} \theta_{d}}{n_{o}^{2}}\right]^{-1 / 2}
\end{aligned}
$$

$\theta_{i}$ and $\theta_{d}$ were the polar angle for incident and diffracted beams. $\sigma$ had wavelength dependence, its effect on the A-O material was more obvious in the short wavelength area $[9,10] . n_{o}$ and $n_{e}$ were the ordinary and extraordinary refractive indices in the direction perpendicularly to the optical axis, respectively. They are the function of the optical wavelength $\lambda_{0}$ in vacuum [10]. Under the momentum-matching condition, the geometry of the wave-vector propagation polar angles can also be expressed [10]. For example, the diffracted polar angle $\theta_{d}$ was related with the incident polar angle $\theta_{i}$ and the optical wavelength $\lambda_{0}$. The acoustic angle $\theta_{a}$ was also determined by $\theta_{i}$ and $\lambda_{0}$.

\section{DESIGN OF THE TeO 2 BASED NARROW BAND AOTF}

For the hyperspectral application, a $\mathrm{TeO}_{2}$ based noncollinear AOTF was designed by using our accurate A-O interaction theory. The geometry of the AOTF was marked in Figure 2.

The main design method of a noncollinear AOTF was as followed: by the A-O interaction theory, the selection of the incident polar angle $\theta_{i}$ was an important and beginning step for the design. The optimum $\theta_{i}$ was connected with many factors (such as, the working wavelength range, the acoustic frequency and the power) [11]. As the optical axis was fixed for certain crystal, the direction of the incident light can be got. The incident face of the AOTF was perpendicular with the incident light. Then the direction of the acoustic wave can be computed by the A-O interaction theory. Further, the position of the piezoelectric transducer could be acquired. The position of the absorber was related with the direction of the acoustic energy flow.

It was worth of mentioning that, the +1 order diffracted beam (which is right-handed elliptically polarized) is utilized in the design. The incident face is not parallel with the exit face (a proper angle exists between them), in order to decrease the shift of the diffracted beam with the scanning optical wavelength [12]. Table 1 listed the design parameters of the AOTF.

\section{THE PERFORMANCE TESTING OF THE AOTF}

By a self-established setup, the performance of the designed narrow-band AOTF was evaluated. A halogen lamp was selected as an incident light source. When the radio frequency (RF) source was on, the piezoelectric transducer bonded on the bottom of $\mathrm{TeO}_{2}$ crystal will generate the acoustic waves launching into the crystal. The light incident on the surface of AOTF can be diffracted with a narrow optical band. The center wavelength of the diffracted light would change with the acoustic frequency. At the outface of the AOTF, the diffracted light was collected by a precise grating spectrometer.

In the experiment, we got the spectrums under a series of the acoustic frequencies $(110,120,130,140,150$, and $160 \mathrm{MHz})$ in Figure 3 . The corresponding diffracted optical central wavelengths were 675.6, 630.0, 590.2, 557.0, 504.3, and $483.3 \mathrm{~nm}$.

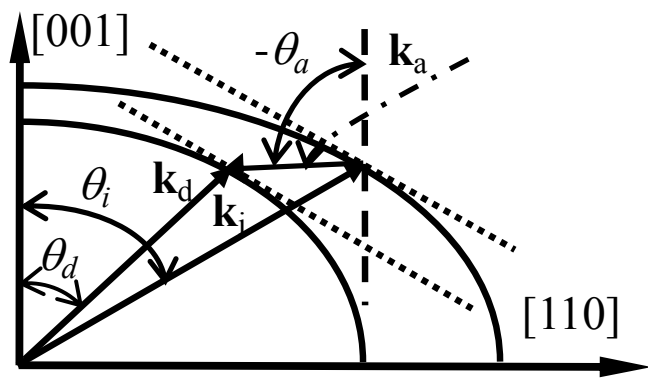

FIG. 1 Wavevector diagram of A-0 action for a noncollinear AOTF.

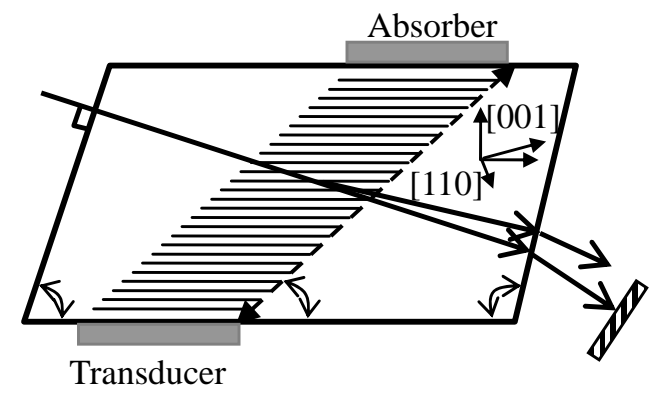

FIG. 2 Design of $\mathrm{a} \mathrm{TeO}_{2}$ crystal for noncollinear AOTF. 

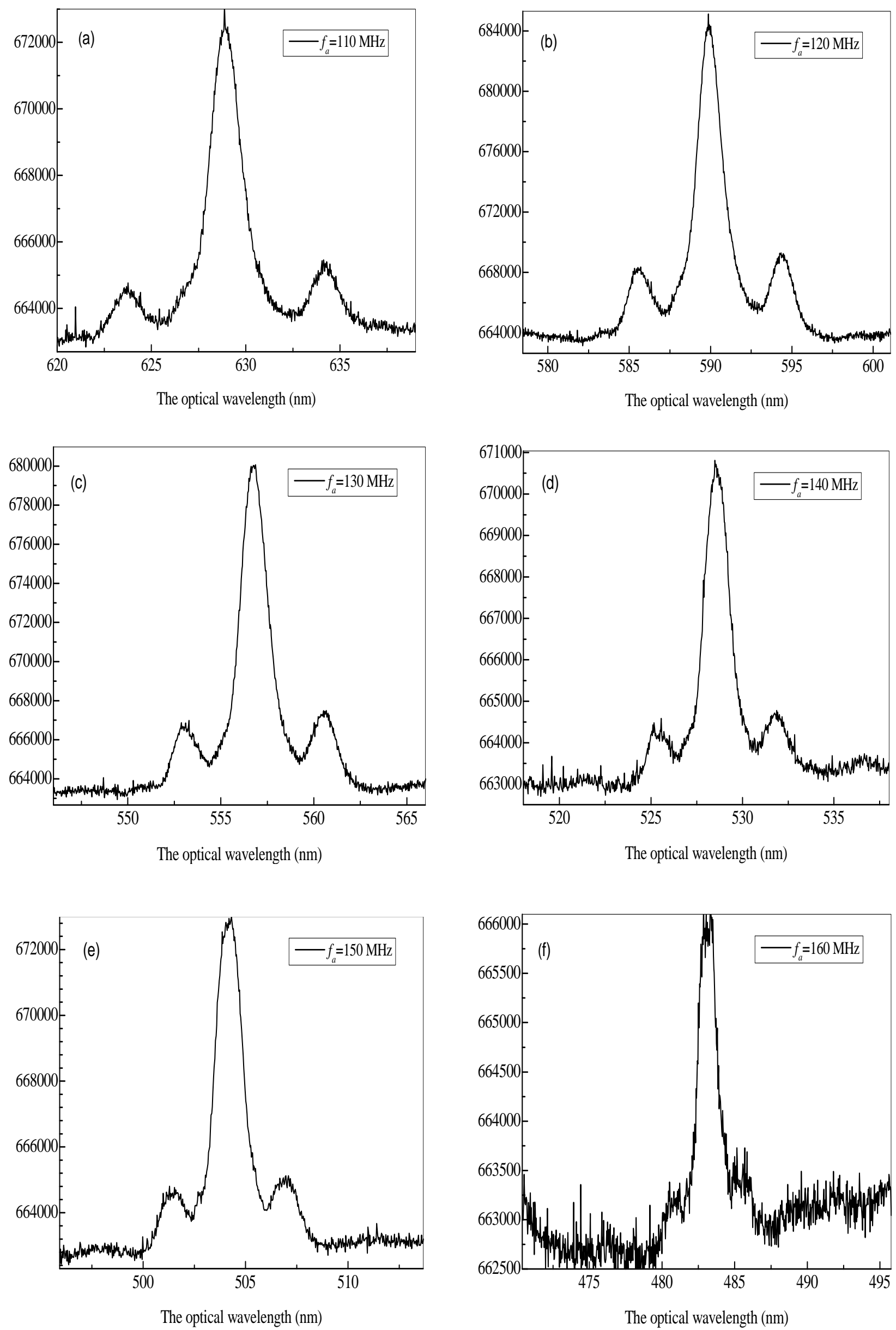

FIG. 3 The spectrums under a series of the acoustic frequency. 


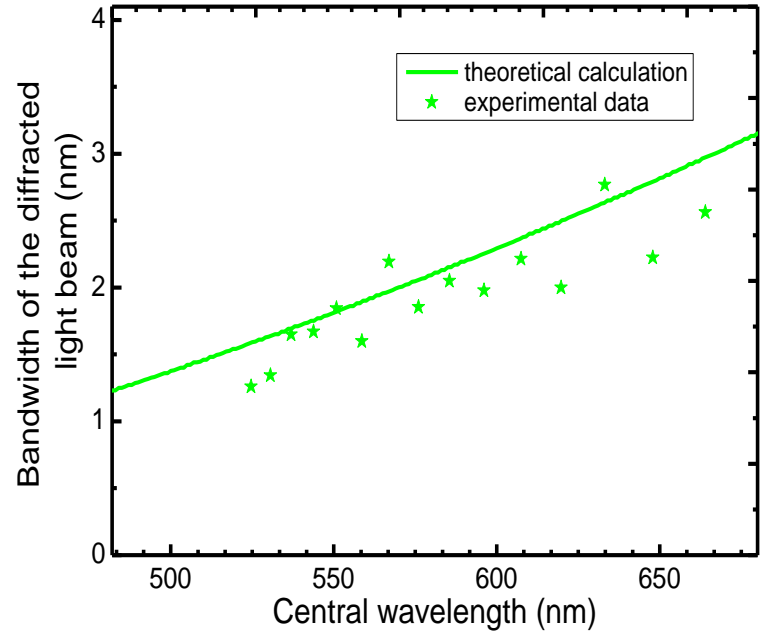

FIG. 4 Relationship between the bandwidth and the central wavelength of the diffracted light.

\begin{tabular}{|c|c|}
\hline Item & $\begin{array}{c}\text { The design parameters and } \\
\text { the performance index }\end{array}$ \\
\hline The acoustic polar angle & $80^{\circ}$ \\
The incident polar angle & $23.8^{\circ}$ \\
The working wave band & $400-700 \mathrm{~nm}$ \\
The tuning range of & $107.5-223.5 \mathrm{MHz}$ \\
the acoustic frequency & $1.0-1.5 \mathrm{~W}$ \\
The acoustic power & $2.85^{\circ} @ 632.80 \mathrm{~nm}$ \\
The incident aperture & $2.9 \mathrm{~nm} @ 632.80 \mathrm{~nm}$ \\
The optical bandwidth & $6.15^{\circ}$ \\
The wedge angle & $6.76 \mathrm{~mm}$ \\
The transducer length & \\
\hline
\end{tabular}

TABLE 1 The design parameters and the performance of the noncollinear AOTF (theoretical computation).

And the bandwidths were 2.51, 2.10, 1.95, 1.80, 1.54, and $1.51 \mathrm{~nm}$ respectively. They were the much narrow bandwidth in the experimental observation with a single $\mathrm{TeO}_{2} \mathrm{AOTF}$, to our knowledge [1, 5, 7, 8, 13]-[15]. The high spectrum resolution of our designed AOTF indicated the excellent ability of spectral analysis. It will be of great significance in hyperspectral imaging and other related areas. Especially, the experimental spectrum results were in good agreement with the theoretical ones (in Figure 4). This demonstrated that our A-O theory of designing AOTF was of significance of keeping the design accuracy. By analysis of the related spectrum datas in the visible range, we found that the bandwidth of the diffracted light increases with the corresponding optical wavelength (in Figure 4). The experimental changing trend of the spectral bandwidth was well accordant with the computation.

Figure 5 gave the tuning relationship between the acoustic frequency and the central optical wavelength of the diffracted light. In Figure 5, the diffracted central wavelength decreased with the increase of the acoustic frequency. The experimental results of the diffracted central wavelength were in accordance with the theoretical calculation at some selected acoustic frequency. It can further indicate the accuracy of our design AOTF.

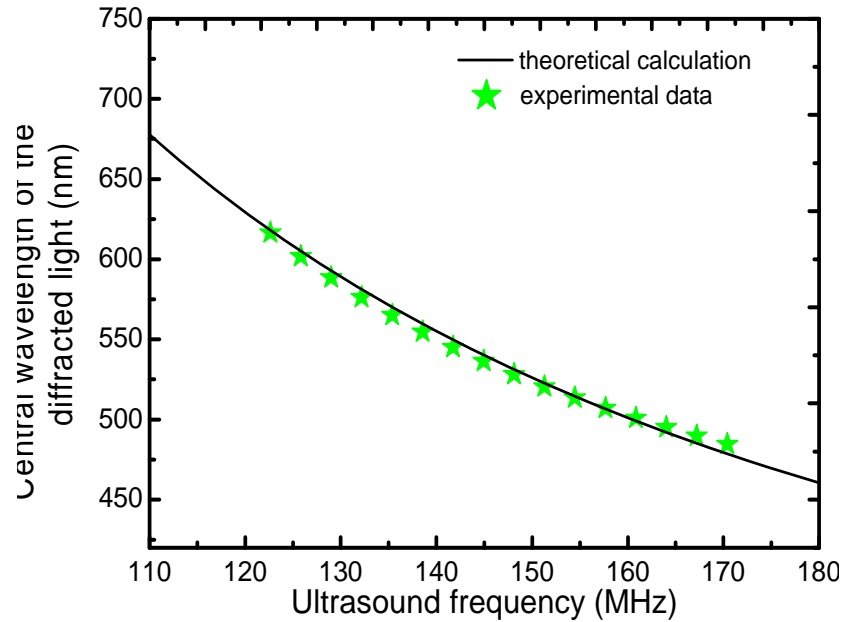

FIG. 5 Relationship between the central wavelength of the diffracted light and acoustic frequency.

\section{THE HYPERSPECTRAL MICROSCOPIC IMAGING EXPERIMENT OF THE RAT SKIN}

\subsection{The experiment system}

The designed AOTF had illuminated good holistic performance from the above testing. And it was advantageous for the hyperspectral imaging applications. In the study, we had selected the self-design AOTF as a spectroscopic element. A hyperspectral microscopic imaging system was established. Figure 6 had shown the configuration of this system. In this system, the trait was the introduction of a convert microscopy. The examples can be place on the object stage of the microscopy, and the AOTF was set at the side exit of the microscopy. This design can make it possible of the biological sample detection. The CCD or the spectrometer was used as the detectors for collecting the diffracted spectrums and images.

\subsection{The hyperspectral microscope imaging of the rat skin}

The biomedical application was an important area for the hyperspectral microscopic imaging system. By our system (in Figure 6), we chose the rat skin tissue as the detected sample. And the hyperspectral imaging experiment was put forward. Figure 7 gave the microscopic images of the rat skin at a series of the acoustic frequency $(125,135,155,160,165$, and $170 \mathrm{MHz}$ ). In Figure 7, every image at certain acoustic frequency was clear. By the scale $(50 \mu \mathrm{m})$ on the images, we can express the better imaging resolution of the system. The stratum of the rat skin (the epidermis and the corium) can be distinguished obviously. And the inner details of the epidermis and the corium can also be shown on the hyperspectral microscopic images. In the epidermis layer, we can see that the keratinocyte cells are arranged periodically, and the elements become more and more compact from stratum corneum to germinal layer. In the corium layer, the glands were shown obviously. And the net like connective group was also displayed (in Figure 8 and Figure 9). At the hyperspectral images, the difference between the glands and the connective group was more obvious than that at the corresponding color im- 


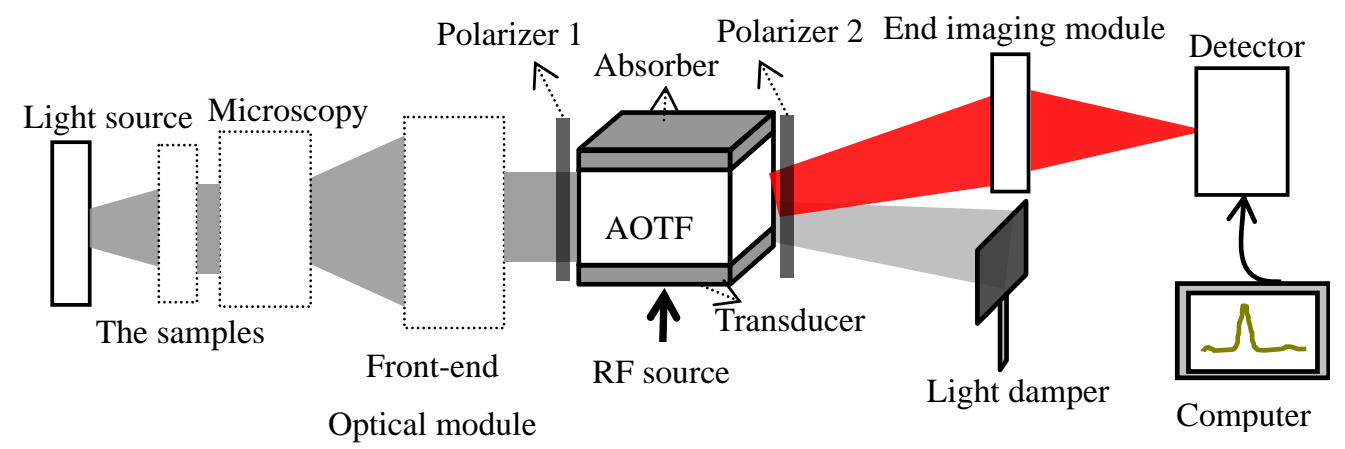

FIG. 6 The noncollinear AOTF based hyperspectral microscopic imaging system.



(a) $f_{a}=125 \mathrm{MHz}$

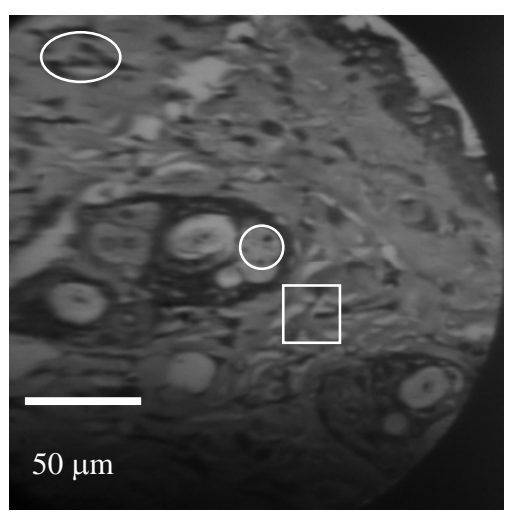

(d) $f_{a}=160 \mathrm{MHz}$



(b) $f_{a}=135 \mathrm{MHz}$

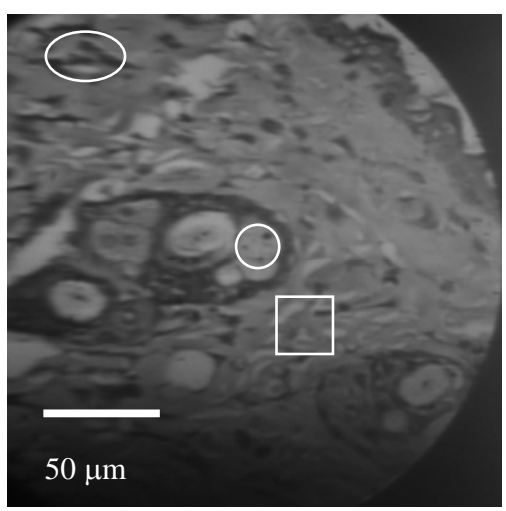

(e) $f_{a}=165 \mathrm{MHz}$

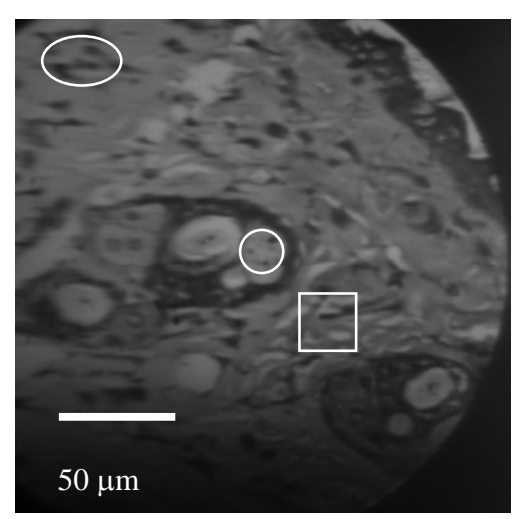

(c) $f_{a}=155 \mathrm{MHz}$

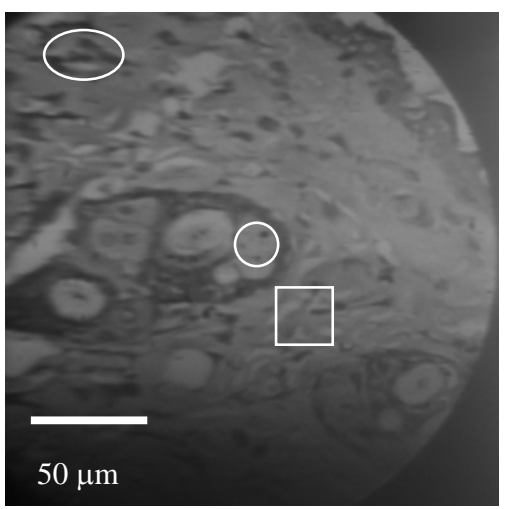

(f) $f_{a}=170 \mathrm{MHz}$

FIG. 7 The hyperspectral microscopic images of the rat skin tissue at a series of acoustic frequencies.



(a) $f_{a}=160 \mathrm{MHz}$

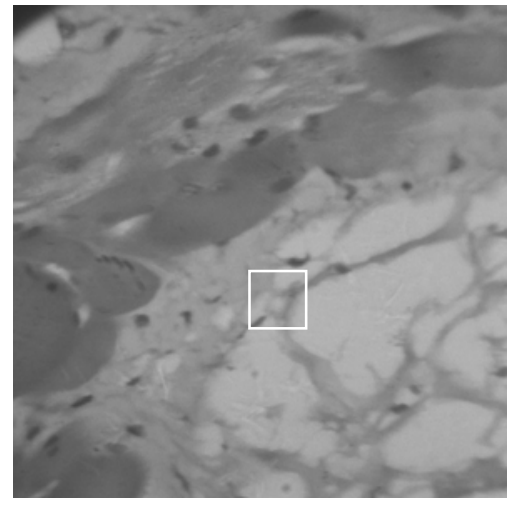

(b) $f_{a}=170 \mathrm{MHz}$

FIG. 8 The part of the corium at the hyperspectral microscopic images. 


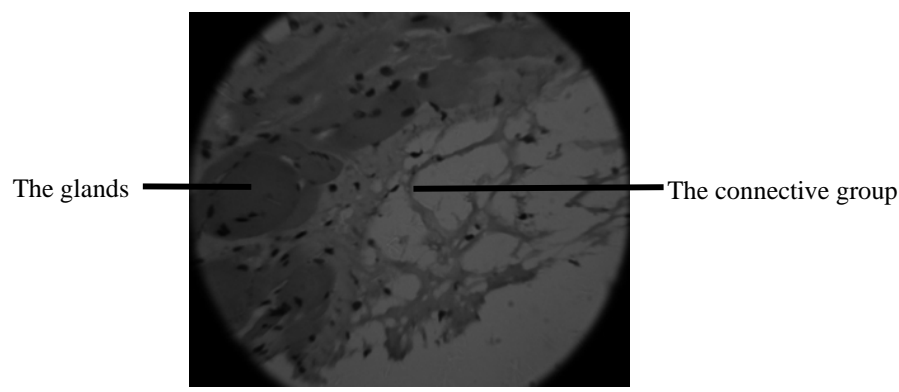

FIG. 9 The color imaging of the rat skin tissue in the corium layer.

age (Figure 9).The cells can be differentiated clearly. Besides, by comparison of the hyperspectal images under the different acoustic frequency, some differences also can be found. For example, in the epidermis, the cells (marked with the solid-line-ellipses) were shown more obvious on the longer wavelength images (Figure $7(\mathrm{a})$ and $7(\mathrm{~b})$ ) than that on the shorter wavelength images Figure $7(\mathrm{c})-(\mathrm{f}))$. In the corium, the inner element of the glands (marked with the circles) appears clearer in the middle wavelength (Figure 7(c) and 7(d)) than that other waveband (Figure 7(a), 7(b), 7(e) and 7(f)). The netlike structure of the connective group (marked with the solidline-squares) was more distinct at the shorter wavelength (as shown in Figure 7(f) and Figure 8(b)).

The study can indicate the applicability and the advantage of our system on biomedicine area. In the future study, the definition of the hyperspectral images would be well improved by introducing a positive and negative lens to end imaging module part (in Figure 6).

\section{CONCLUSION}

In this letter, the authors designed a narrow-band noncollinear VIS AOTF using a single $\mathrm{TeO}_{2}$ crystal by comprehensively considering the related property of the interaction material. A functional hyperspectral microscopic imaging system was designed on the basis of the self-designed noncollinear AOTF. The introduction of a convert microscopy made the system more applicable in the biomedical area. In the study, the conclusion was that:

1. The performance testing results indicated the designed AOTF had famous capability. The spectral resolution in the visible range was high, and it was much significant for the hyperspectral applications especially.

2. In the performance testing experiment, the results (such as the spectrums of the diffracted light, the tuning relationship between the central diffracted optical wavelength and the acoustic frequency) were acquired. It was worth mentioning that, the acquired results and the rules (experimentally) were in accordance with the theoretical prospects. Our A-O theory was identified to be applicable and valid for the $\mathrm{TeO}_{2}$ noncollinear AOTF design.

3. The hyperspectral microscopic imaging system was established. The experimental results of the rat skin in visible range had indicated the feasibility of the system in the biomedical study. In the study, the stratum of the rat skin can be distinguished obviously. The inner details in the skin tissue (such as stratum corneum, germinal layer, connective group, and etc.) can also been differentiated clearly. The AOTF based hyperspectral microscopic imaging system had exhibited good imaging performance.

\section{ACKNOWLEDGEMENTS}

The authors acknowledge the support from National Natural Science Foundation of China under Grant (No. 61107092) and Program for Changjiang Scholars and Innovative Research Team in University (No. IRT1115) and Program for the Excellent Teacher of Fujian Normal University (No. fjsdjk2012055).

\section{References}

[1] N. Gupta, R. Dahmani, and S. Choy, "Acousto-optic tunable filter based visible-to near-infrared spectropolarimetric imager," opt. Eng. 41, 1033-1038 (2002).

[2] V. B. Voloshinov, K. B. Yushkov, and B. B J. Linde, "Improvement in performance of a $\mathrm{TeO}_{2}$ acousto-optic imaging spectrometer," J. Opt. A 9, 341-347 (2007).

[3] Y. Yuan, J. Y. Hwang, M. Krishnamoorthy, K. Ye, Y. Zhang, J. Ning, R. C. Wang, et. al. "High-throughput acousto-optic-tunablefilter-based time-resolved fluorescence spectrometer for optical biopsy," Opt. Lett. 34, 1132-1134 (2009).

[4] S. Y. Ryu, J. W. You, Y. Kwak, and S. Kim, "Design of a prism to compensate the angular shift error of the acousto-optic tunable filter," Opt. Express 16, 17138-17147 (2008).

[5] J. W. You, J. Ahn, S. Kim, and D. Kim, "Efficient double-filtering with a single acoustooptic tunable filter," Opt. Express 16, 21505-21511 (2008).

[6] K. B. Yushkov, S. Dupont, J. C. Kastelik and V. B. Voloshinov, "Polarization-independent imaging with an acousto-optic tandem system," Opt. Lett. 35, 1416-1418 (2010).

[7] I. C. Chang, "Noncollinear acousto-optic filter with large angular aperture," Appl. Phys. Lett. 25, 370-372 (1974).

[8] P. A. Gass, and J. R. Sambles, "Accurate design of noncollinear acousto-optic tunable filter," Opt. Lett. 16, 429-431 (1991).

[9] N. Uchida, “Optical properties of single-crystal paratellurite $\left(\mathrm{TeO}_{2}\right)$," Phys. Rev. B 4, 3736-3745 (1971).

[10] C. Zhang, Z .Zhang, Y. Yang, and H. Wang, "The design and analysis of a noncollinear acousto-optic tunable filter," Opt. Lett. 32, 2417-2419 (2007).

[11] C. Zhang, Z. Zhang, H. Wang, and Y. Yang, "Analysis of the optimum optical incident angle for an imaging acousto-optic tunable filter," Opt. Express 15, 11883-11888 (2007).

[12] D. R. Suhre, and J. G. Throdore, "White-light imaging by use of a multiple passband acousto-optic tunable filter," Appl. 0pt. 35, 4494-4501 (1996).

[13] N. Gupta, and V. B. Voloshinov, "Development and characterization of two transducer imaging acousto-optic tunable filters with extended tuning range," Appl. Opt. 46, 1081-1088 (2007).

[14] http://www.auniontech.com/p/ao_modulator_aotf/A0_Tunable_ Filter.html

[15] http://www.brimrose.com/cn/products/acousto_optics.html 